Pacific Journal of Mathematics

THE REDUCING IDEAL IS A RADICAL 


\title{
THE REDUCING IDEAL IS A RADICAL
}

\author{
T. W. PALMer
}

For any *-algebra $\mathfrak{A}$ the reducing ideal $\mathfrak{A}_{R}$ of $\mathfrak{A}$ is the intersection of the kernels of all the *-representations of $\mathfrak{U}$. Although the reducing ideal has been called the *-radical, and obviously satisfies $\left(\mathfrak{H} / \mathfrak{A}_{R}\right)_{R}=\{0\}$, it has not previously been shown to satisfy another of the fundamental properties of an abstract radical except in the case of hermitian Banach *-algebras where it equals the Jacobson radical. In this paper we prove two extension theorems for *-representations. The more important one states that any essential *-representation of a $*$-ideal of a $U^{*}$-algebra (a fortiori, of a Banach *-algebra) has a unique extension to a *-representation of the whole algebra. These theorems show in particular that $\left(\mathfrak{A}_{R}\right)_{R}=\mathfrak{X}_{R}$ if $\mathfrak{A}$ is either a commutative *-algebra or a $U^{*_{-}}$ algebra. The somewhat stronger statements which are actually proved, together with previously known properties of the reducing ideal, show that the reducing ideal defines a radical subcategory of each of the following three semi-abelian categories:

(1) Commutative *algebras and *homomorphisms.

(2) Banach *-algebras and continuous *-homomorphisms.

(3) Banach *-algebras and contractive *-homomorphisms.

The concept of the reducing ideal was introduced by Gelfand and Naimark in their classic paper [2, p. 463]. It has subsequently been studied by Kelley and Vaught [5, p. 51] and the present author $[7$, p. 63] and [8, p. 930]. The concept is discussed in [10, pp. 210, 226] and [6, p. 259]. In [11, 1479] Yood gave a definition of the *-radical which agrees with our definition for Banach *-algebras but differs for certain other types of *-algebras.

Our main extension theorem (3.1, below) was previously known for $B^{*}$-algebras [1, Proposition 2.10.4]. It has a number of applications besides the one discussed here. For example it immediately implies the conclusion of [4, Theorem 23] with hypotheses weaker than those of [4, Theorem 22].

In $\S 1$ we give necessary background information. The case of commutative *-algebras is considered in $\S 2$ and of $U^{*}$-algebras in $\S 3$. The category theory results are described in $\S 4$ where we use the terminology of M. Gray [3] for the general theory of radicals.

In general we follow the terminology, of Rickart's book [10]. Further details and related results will be found in the author's forthcoming monograph [9]. 
1. Definitions and preliminary results. We review some basic definitions and results for the convenience of the reader and in order to fix notation. Throughout this paper all algebras and linear spaces will have the complex field $C$ as scalar field unless the real field is explicitly specified. No other scalar field is considered. The complex conjugate of $\lambda \in C$ will be denoted by $\lambda^{*}$.

An involution on an algebra $\mathfrak{X}$ is a conjugate linear, anti-multiplicative, involutive map of $\mathfrak{A}$ onto itself. A *algebra is an algebra together with a fixed involution which will always be denoted by $\left(^{*}\right)$. A subset of a ${ }^{*}$-algebra is called a ${ }^{*}$-subset iff it closed under the involution. A map between *-algebras is called a *-map iff it preserves their involutions (i.e. $\left.\varphi\left(a^{*}\right)=\varphi(a)^{*}\right)$. A *-representation $T$ of a *algebra is a ${ }^{*}$-homomorphism (i.e. an algebra homomorphism which is also a *-map) into the *-algebra $\left[\mathfrak{S}_{T}\right]$ of all bounded linear operators on some Hilbert space $\mathfrak{S}_{T}$. The meaning of each more specific term with a *-prefix (e.g. *-subalgebra, *-isomorphism) follows from these definitions. In particular a Banach *-algebra is simply a *-algebra with a norm relative to which it is a Banach algebra. No relationship between the involution and norm is postulated.

We review briefly the standard Gelfand-Naimark construction of *-representations from positive linear functionals since later proofs depend intimately on this material (cf. [2], [6], [9] or [10]). A linear functional $\omega$ on a ${ }^{*}$-algebra $\mathfrak{U}$ is called positive iff

$$
\omega\left(a^{*} a\right) \geqq 0
$$

$\forall a \in \mathfrak{A}$.

For any positive linear functional $\omega$ denote the left ideal

$$
\left\{a \in \mathfrak{X}: \omega\left(a^{*} a\right)=0\right\}=\left\{a \in \mathfrak{X}: \omega\left(b^{*} a\right)=0, \forall b \in \mathfrak{X}\right\}
$$

by $\mathfrak{N}_{\omega}$. Let

$$
\mathfrak{U}^{\omega}=\mathfrak{U} / \mathfrak{U}_{\omega} \text { • }
$$

For each $a \in \mathfrak{A}$ let $a^{\omega}$ be the image $a+\mathfrak{A}_{\omega}$ of $a$ in $\mathfrak{X}^{\omega}$. Then for all $a^{\omega}, b^{\omega} \in \mathfrak{Z}^{\omega}$

$$
\left(a^{\omega}, b^{\omega}\right)=\omega\left(b^{*} a\right)
$$

is well defined and gives $\mathfrak{A}^{\omega}$ the structure of a pre-Hilbert space (i.e. a possibly incomplete inner-product space). The left regular representation of $\mathfrak{A}$ on itself induces a ${ }^{*}$-homomorphism $\widetilde{T}^{\omega}$ of $\mathscr{U}$ into the *-algebra of all (not necessarily bounded) linear operators on $\mathfrak{Z}^{\omega}$ which have adjoints on $\mathfrak{X}^{\omega}$. The positive linear functional $\omega$ is called admissible iff the range of $\widetilde{T}^{\omega}$ consists of bounded operators so that $\widetilde{T}^{\omega}$ induces a ${ }^{*}$-representation $T^{\omega}$ of $\mathfrak{A}$ on the Hilbert space completion $\mathfrak{X}^{\omega^{-}}$of $\mathfrak{X}^{\omega}$. 
An admissible positive linear functional $\omega$ is called representable iff there is some ${ }^{*}$-representation $T$ and some topologically cyclic vector $x \in \mathfrak{S}_{T}$ for $T$ such that

$$
\omega(a)=\left(T_{a} x, x\right) \quad \forall a \in \mathfrak{X} .
$$

The set of representable positive linear functionals on a *algera $\mathfrak{X}$ will be denoted by $R(\mathfrak{Y})$. For each nonzero $\omega$ in $R(\mathfrak{U})$

$$
|\omega|=\sup \left\{\omega(a)^{2} / \omega\left(a^{*} a\right): a \in \mathfrak{X} \sim \mathfrak{U}_{\omega}\right\}
$$

is finite. For the zero linear functional, which always belongs to $R(\mathfrak{U})$, we set $|0|=0$. For each $\omega \in R(\mathfrak{X})$ there is a unique vector $x_{\omega}$ in $\mathfrak{2}^{\omega^{-}}$such that

$$
T_{a}^{\omega} x_{\omega}=a^{\omega}
$$

$\forall a \in \mathfrak{N}$.

[9, Theorem 1.4.8]. This vector is a topologically cyclic vector for $T^{\omega}$ which also satisfies

$$
\left\|x_{\omega}\right\|^{2}=|\omega| \text { and } \omega(a)=\left(T_{a}^{\omega} x_{\omega}, x_{\omega}\right) \quad \forall a \in \mathfrak{A} .
$$

For a *-algebra $\mathfrak{A}$ let

$$
R_{1}(\mathfrak{Z})=\{\omega \in R(\mathfrak{Z}):|\omega| \leqq 1\} .
$$

A linear functional $\omega$ on $\mathfrak{A}$ is called a state iff $\omega \in R(\mathfrak{V})$ and $|\omega|=1$. A linear functional $\omega \in R(\mathfrak{R})$ is called pure iff $\omega=\omega_{1}+\omega_{2}$ with $\omega_{1}, \omega_{2} \in R(\mathfrak{V})$ implies that $\omega_{1}$ and $\omega_{2}$ are (nonnegative real) multiples of $\omega$. Let $P(\mathfrak{O})$ denote the set of pure states of 2 . Then $P(\mathfrak{O}) \cup\{0\}$ is the set of extreme points of the convex set $R_{1}(\mathfrak{R})$.

If $\mathfrak{A}$ is a Banach *algebra it is well known that $R_{1}(\mathfrak{Z})$ is compact in the $\mathfrak{2}$-topology. Thus $R_{1}(\mathfrak{Z})$ is the closed convex hull of $P(\mathfrak{O}) \cup\{0\}$ by the Krein-Milman theorem. If $\mathfrak{A}$ is an arbitrary *-algebra (e.g. \{complex polynomials\} with conjugation of coefficients as the involution) then $R_{1}(\mathfrak{X})$ need not be compact.

Lemma 1.1. If $\mathfrak{A}$ is any *algebra, $R_{1}(\mathfrak{C})$ is the closed convex hull of $P(\mathfrak{Z}) \cup\{0\}$.

Proof. For any $\omega \in R_{1}(\mathfrak{Z})$ let

$$
\mathfrak{S}_{\omega}=\left\{\omega^{\prime} \in R_{1}(\mathfrak{Z}):\left\|T_{a}^{\omega^{\prime}}\right\| \leqq\left\|T_{a}^{\omega}\right\| \text { for all } a \in \mathfrak{X}\right\} \text {. }
$$

A slight adaptation of a well known proof $\left[10\right.$, p. 222] shows that $\mathfrak{\subseteq}_{\omega}$ is compact and convex [9, Proposition 1.5.6]. Similarly one can adapt another well known proof [10, p. 225] to show that the set of extreme points of $\mathfrak{S}_{\omega}$ is $\{0\} \cup\left(\Im_{\omega} \cap P(\mathfrak{2})\right)$ [9, Proposition 1.6.6]. Thus $\mathfrak{S}_{\omega}=$ $\overline{\mathrm{co}}\left(\{0\} \cup\left(\mathfrak{S}_{\omega} \cap P(\mathfrak{Z})\right)\right)$. Therefore 


$$
\begin{aligned}
& R_{1}(\mathfrak{X})=\bigcup_{\omega \in R_{1}(\mathfrak{x})} \mathfrak{S}_{\omega}=\bigcup_{\omega \in R_{1}(\mathfrak{x})} \overline{\mathrm{co}}(\{0\} \cup P(\mathscr{R})) \\
& \subseteq \overline{\mathrm{co}}(\{0\} \cup P(\mathfrak{X})) \subseteq R_{1}(\mathfrak{X}) \text {. }
\end{aligned}
$$

LEMMA 1.2. Let $\mathfrak{A}$ be $a^{*}$-algebra and let $\omega \in R(\mathfrak{U})$. The following are equivalent.

(a) $\omega$ is pure.

(b) $T^{\omega}$ is topologically irreducible.

(c) The set $\left(T^{\omega}\right)^{\prime}$ of operators in $\left[\mathfrak{A}^{\omega^{-}}\right]$which commute with $T_{a}^{\omega}$ for each $a \in \mathfrak{A}$ is the set of complex multiples of the identity.

Proof. [10, p. 211 and 223], [9, Theorems 1.6.1 and 1.6.5].

Definition 1.3. For any *-algebra $\mathfrak{A}$ the reducing ideal of $\mathfrak{A}$ is denoted by $\mathfrak{U}_{R}$ and defined by

$$
\mathfrak{U}_{R}=\bigcap\left\{\operatorname{Ker}(T): T \text { is a }{ }^{*} \text {-representation of } \mathfrak{U}\right\} \text {. }
$$

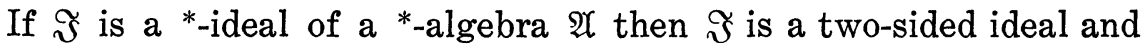
$\mathfrak{U} / \mathfrak{\Im}$ is a ${ }^{*}$-algebra in an obvious sense.

Proposition 1.4. Let $\mathfrak{A}$ be $a^{*}$-algebra. Then the reducing ideal $\mathfrak{A}_{R}$ of $\mathfrak{A}$ is a ${ }^{*}$-ideal which equals:

$\bigcap\{\operatorname{Ker}(T): T$ is a topologically irreducible *-representation of $\mathfrak{Q}\}$

$=\bigcap\left\{\operatorname{Ker}\left(T^{\omega}\right): \omega \in R(\mathfrak{Q})\right\}=\bigcap\left\{\operatorname{Ker}\left(T^{\omega}\right): \omega \in P(\mathfrak{R})\right\}$

$=\bigcap\left\{\mathbb{R}_{\omega}: \omega \in R(\mathfrak{R})\right\}=\bigcap\left\{\mathbb{R}_{\omega}: \omega \in P(\mathfrak{R})\right\}$

$=\{a \in \mathfrak{X}: \omega(a)=0, \forall \omega \in R(\mathfrak{X})\}=\{a \in \mathfrak{X}: \omega(a)=, \forall \omega \in P(\mathfrak{X})\}$.

Furthermore $\left(\mathfrak{U} / \mathfrak{U}_{R}\right)_{R}=\{0\} . \quad$ If $\mathfrak{X}$ is a Banach ${ }^{*}$-algebra then $\mathfrak{X}_{R}$ is closed so that $\mathfrak{2} / \mathfrak{Q}_{R}$ is a Banach *-algebra.

Proof. Use Lemma 1.1 to adapt the proof of [10, Theorem 4.4.10]. For details and further results see [9, Theorem 1.7.2 and 1.7.5].

Lemma 1.1 and this proposition do not seem to have been noted previously in this degree of generality. However they were essentially known.

We now turn to the theory of $U^{*}$-algebras. For additional information see [7], [8], or [9].

If $\mathfrak{A}$ is a *-algebra without an identity let $\mathfrak{X}^{1}$ denote the *algebra with identity which has $\boldsymbol{C} \oplus \mathfrak{A}$ as underlying linear space and in which the multiplication and involution are defined by $(\lambda \oplus a)(\mu \oplus b)=$ $\lambda \mu \oplus(\lambda b+\mu a+a b)$ and $(\lambda \oplus a)^{*}=\lambda^{*} \oplus a^{*}$ for all $\lambda, \mu \in C$ and all $a, b \in \mathfrak{A}$. We regard $\mathfrak{X}$ as embedded in $\mathfrak{X}^{1}$ by the map $a \rightarrow 0 \oplus a$. If $\mathfrak{A}$ already has an identity let $\mathfrak{A}^{1}=\mathfrak{N}$. In either case we write $\lambda+a$ 
for $\lambda 1+a$ where 1 is the identity of $\mathfrak{X}^{1}$. Then, for instance, the spectrum of an element in $\mathfrak{A}$ is the same with relation to $\mathfrak{X}$ or $\mathfrak{X}^{1}$. Furthermore the Jacobson radical of $\mathfrak{X}$ and $\mathfrak{X}^{1}$ agree and the reducing ideal of $\mathfrak{X}$ and $\mathfrak{U}^{1}$ agree.

Definition 1.5. A *-algebra $\mathfrak{A}$ is called a $U^{*}$-algebra iff $\mathfrak{A}$ is contained in the linear span of the set $\mathfrak{X}_{U}^{1}$ of unitary elements in $\mathfrak{Q}^{1}$. If $\mathfrak{X}$ is a $U^{*}$-algebra and $a \in \mathfrak{A}$ then

$$
\boldsymbol{\nu}_{\mathfrak{x}}(a)=\inf \left\{\sum_{j=1}^{n} \lambda_{j}: a=\sum_{j=1}^{n} \lambda_{j} u_{j} \text { where } n \in N, \lambda_{j} \in C \text {, and } u_{j} \in \mathfrak{U}_{U}^{1}\right\} .
$$

Lemma 1.6. Let $\mathfrak{X}$ be a $U^{*}$-algebra. Then $\nu_{\mathfrak{x}}$ is an algebra pseudo-norm, (i.e. $\nu_{\mathfrak{x}}(\lambda a)=|\lambda| \nu_{\mathfrak{x}}(a), \nu_{\mathfrak{x}}(a+b) \leqq \nu_{\mathfrak{x}}(a)+\nu_{\mathfrak{u}}(b), \nu_{\mathfrak{x}}(a b) \leqq$ $\nu_{\mathfrak{r}}(a) \nu_{\mathfrak{q}}(b)$ for all $\left.a, b \in \alpha\right)$.

Proof. Obvious.

For any *-algebra $\mathfrak{A}$ let

$$
\mathfrak{U}_{q U}=\left\{v \in \mathfrak{X}: v^{*} v=v v^{*}=v+v^{*}\right\}
$$

be the set of quasi-unitary elements in $\mathfrak{A}$. For any subset $\mathfrak{S}$ of $\mathfrak{A}$ let $\mathfrak{S}^{U}$ be the linear span of $\mathfrak{S} \cap \mathfrak{A}_{q U}$.

Lemma 1.7. Let $\mathfrak{X}$ be $a{ }^{*}$-algebra. Then $\mathfrak{Q}^{U}$ is a $a^{*}$-subalgebra of $\mathfrak{A}$ which is a $U^{*}$-algebra. Furthermore $\mathfrak{X}^{U}$ contains every ${ }^{*}$-subalgebra of $\mathfrak{A}$ which is a $U^{*}$-algebra. In particular $\mathfrak{A}$ is a $U^{*}$-algebra iff $\mathfrak{U}=\mathfrak{U}^{U}$. In this case

$\boldsymbol{\nu}_{\mathfrak{x}}(a)=\inf \left\{\sum_{j=1}^{n} \lambda_{j}: a=\sum_{j=1}^{n} \lambda_{j} v_{j}, 0=\sum_{j=1}^{n} \lambda_{j}\right.$ where $n \in N, \lambda_{j} \in C$ and $\left.v_{j} \in \mathfrak{U}_{q U}\right\}$.

Finally if $\mathfrak{\Im}$ is a one-or two-sided ideal in $\mathfrak{A}$ then $\mathfrak{\Im}^{U}$ is $a^{*}$-ideal in भ.

Proof. Straightforward or see [8] or [9].

LEMmA 1.8. Let $\mathfrak{X}$ be a $U^{*}$-algebra and let $\mathfrak{B}$ be $a{ }^{*}$-algebra. Let $\varphi: \mathfrak{A} \rightarrow \mathfrak{B}$ be $a^{*}$-homomorphism. Then $\varphi(\mathfrak{Z})$ is a $U^{*}$-algebra and $\boldsymbol{\nu}_{\varphi(\mathfrak{r})}(\varphi(a)) \leqq \boldsymbol{\nu}_{\mathfrak{x}}(a)$ for all $a \in \mathfrak{N}$. Furthermore if $\mathfrak{B}$ is the algebra of all (not necessarily bounded) linear operators with adjoints on a preHilbert space, then $\varphi(\mathfrak{Z})$ is contained in the set of bounded operators and $\|\varphi(a)\| \leqq \nu_{\mathfrak{x}}(a)$ for all $a \in \mathfrak{N}$.

Proof. This follows directly from Lemma 1.7 or see [7], [8] or 
[9].

By slight abuse of language we call a *homomorphism into the type of *-algebra described in the last sentence of Lemma 1.8 a *-representation on a pre-Hilbert space. When the range of such a map consists of bounded operators we call it a normed *-representation on a pre-Hilbert space. (Of course any *-representation of any *-algebra (by definition, on a Hilbert space) is automatically normed [10, p. 205] or [9, Corollary 1.2.4].)

COROLLARY 1.9. Every *-representation of a $U^{*}$-algebra on a pre-Hilbert space is normed. Every positive linear functional on a $U^{*}$-algebra is admissible. A positive linear functional on a $U^{*}$-algebra $\mathfrak{A}$ is representable iff it is the restriction of some positive linear functional on $\mathfrak{2}^{1}$.

Proof. For the last sentence see [10, p. 218] or [9, Theorem 1.4.8].

Definition 1.10. Let $\mathfrak{A}$ be a ${ }^{*}$-algebra. For any $a \in \mathfrak{A}$ let $\gamma_{\mathfrak{x}}(a)=\sup \left\{\left\|T_{a}\right\|: T\right.$ is a ${ }^{*}$-representation of $\mathfrak{A}$ on a Hilbert space $\}$.

It is not hard to show [9, Theorem 2.1.2] that $\gamma_{\mathfrak{x}}(a)=\sup \left\{\left\|T_{a}\right\|\right.$ : $T$ is a topologically irreducible *-representation of $\mathfrak{A}$ on a Hilbert space $\}=\sup \left\{\omega\left(a^{*} a\right)^{1 / 2}: \omega \in R_{1}(\mathfrak{R})=\sup \left\{\omega\left(a^{*} a\right)^{1 / 2}: \omega \in P(\mathfrak{X})\right\}\right.$. In a perfectly general *-algebra $\gamma_{\mathfrak{r}}(a)=\infty$ is possible. However if $\gamma_{\mathfrak{r}}$ is finite valued then it is the largest algebra pseudonorm on $\mathfrak{A}$ which satisfies the $B^{*}$-condition: $\gamma_{\mathfrak{r}}\left(a^{*} a\right)=\gamma_{\mathfrak{r}}(a)^{2}$ for all $a \in \mathfrak{N}$. We call $\gamma_{\mathfrak{r}}$ the GelfandNaimark pseudo-norm on $\mathfrak{A}$. Note that $\mathfrak{A}_{R}=\left\{a \in \mathfrak{A}: \gamma_{\mathfrak{r}}(a)=0\right\}$.

COROLlary 1.11. If $\mathfrak{A}$ is a $U^{*}$-algebra then

$$
\gamma_{\mathfrak{U}}(a) \leqq \nu_{\mathfrak{H}}(a)
$$

for all $a \in \mathfrak{R}$.

Proof. Obvious from Lemma 1.8.

THEOREM 1.12. Let $\mathfrak{A}$ be a Banach *-algebra. Then $\mathfrak{A}$ is a $U^{*}$ algebra and $\gamma_{\mathfrak{x}}=\nu_{\mathfrak{x}}$.

Proof. [7, Theorem 4] or [9, Theorem 3.1.12].

2. Commutative *-algebras. We are now in a position to treat this case easily. Several of our results are essentially known but are 
usually stated in less generality.

TheOREM 2.1. Let $\mathfrak{A}$ be a commutative *algebra. Then $P(\mathfrak{X})$ is the set of *-homomorphisms of $\mathfrak{X}$ onto $\boldsymbol{C}$.

Proof. Suppose $\omega$ is a pure state. Then $\left(T^{\omega}\right)^{\prime}=C I$ by Lemma 1.2 where $I$ is the identity operator in $\left[\mathfrak{H}^{\omega^{-}}\right]$. Since $\mathfrak{A}$ is commutative $T_{\mathfrak{\vartheta}}^{\omega} \subseteq\left(T_{\mathfrak{\vartheta}}^{\omega}\right)^{\prime}$. Since $\omega \neq 0, T^{\omega} \neq 0$ so $T_{\mathfrak{\imath}}^{\omega}=C I$. Let $T_{a}^{\omega}=$ $\varphi(a) I$ for all $a \in \mathfrak{A}$. Then $\varphi$ is a ${ }^{*}$-homomorphism of $\mathfrak{A}$ onto $C$ and $\omega(a)=\left(T_{a}^{\omega} x_{\omega}, x_{\omega}\right)=\left(\varphi(a) x_{\omega}, x_{\omega}\right)=\varphi(a)|\omega|=\varphi(a)$ for all $a \in \mathfrak{R}$. Thus $\omega=\varnothing$ is a ${ }^{*}$-homomorphism of $\mathfrak{U}$ onto $\boldsymbol{C}$.

Conversely suppose $\omega$ is a ${ }^{*}$-homomorphism of $\mathfrak{X}$ onto $\boldsymbol{C}$. Then $\omega\left(a^{*} a\right)=\omega\left(a^{*}\right) \omega(a)=|\omega(a)|^{2}$ for all $a \in \mathfrak{A}$ so that $\omega$ is a state. The map $a^{\omega} \rightarrow \omega(a)$ for all $a \in \mathfrak{N}$ is a linear isometry of $\mathfrak{X}^{\omega}$ onto $C$. Thus $\mathfrak{H}^{\omega}=\mathfrak{X}^{\omega^{-}}$is linearly isometric to $\boldsymbol{C}$ so that $T^{\omega} \neq 0$ is irreducible. Therefore $\omega$ is a pure state by Lemma 1.2.

Corollary 2.2. Let $\mathfrak{A}$ be a commutative *algebra. For each $a \in \mathfrak{X}$ let $\check{a}: P(\mathfrak{H}) \rightarrow C$ be defined by $\grave{a}(\omega)=\omega(a)$ for all $\omega \in P(\mathfrak{Z})$. Let $P(\mathfrak{Z})$ carry the weakest topology which makes each à continuous. Let $C_{\infty}(P(\mathfrak{Y}))$ be the set of continuous but not necessarily bounded complex valued functions on $P(\mathfrak{X})$. Then $P(\mathfrak{H})$ is Tychonoff space and

$$
\text { (^): } \mathfrak{X} \longrightarrow C_{\infty}(P(\mathfrak{X}))
$$

is a*-homomorphism with kernel $\mathfrak{A}_{R}$.

Proof. Immediate from Theorem 2.1 and Proposition 1.4.

THEOREM 2.3. Let $\mathfrak{U}$ be a commutative *-algebra. Let $\mathfrak{B}$ be a ${ }^{*}$-ideal of $\mathfrak{X}$ and let $\Im$ be $a^{*}$-ideal of $\mathfrak{B}$. For each $\omega \in P(\Im)$ there is an $\bar{\omega} \in P(\mathfrak{I})$ such that $\omega$ is the restriction of $\bar{\omega}$.

Proof. Theorem 2.1 shows that $\omega$ is a *homomorphism of $\mathfrak{\Im}$ onto $C$. Let $e \in \mathfrak{F}$ satisfy $\omega(e)=1$. We may assume $e=e^{*}$ since $\omega$ is a *-map. For any $a \in \mathfrak{A}, e a \in \mathfrak{B}$ so $e^{2} a \in \mathfrak{\Im}$. Define $\bar{\omega}(a)=\omega\left(e^{2} a\right)$ for all $a \in \mathfrak{N}$. Then $\bar{\omega}$ is clearly linear and if a, $b \in \mathfrak{A}$ then $\bar{\omega}(a b)=$ $\omega\left(e^{2} a\right)=\omega(e)^{2} \omega\left(a e^{2} b\right)=\omega\left(e^{2} a e^{2} b\right)=\omega\left(e^{2} a\right) \omega\left(e^{2} b\right)=\bar{\omega}(a) \bar{\omega}(b)$, and $\bar{\omega}\left(a^{*}\right)=$ $\omega\left(e^{2} a^{*} b\right)=\omega\left(e^{2} a\right)^{*}=\bar{\omega}(a)^{*}$. Thus $\bar{\omega}$ is a ${ }^{*}$-homomorphism of $\mathfrak{U}$ onto $\boldsymbol{C}$ and thus by Theorem 2.1 $\bar{\omega} \in P(\mathfrak{H})$. If $a \in \mathfrak{\Im}$ then $\bar{\omega}(a)=\omega\left(e^{2} a\right)=$ $\omega(e)^{2} \omega(a)=\omega(a)$. Thus $\bar{\omega}$ satisfies the theorem.

CoROLlaRY 2.4. Let $\mathfrak{A}$ be a commutative *algebra. Let $\Im$ be a *-ideal of $\mathfrak{N}_{R}\left(e . g . a^{*}\right.$-ideal of $\mathfrak{X}$ included in $\left.\mathfrak{N}_{R}\right)$. Then $\mathfrak{\Im}_{R}=\mathfrak{\Im}$. In 
particular $\left(\mathfrak{U}_{R}\right)_{R}=\mathfrak{A}_{R}$.

Proof. If $\mathfrak{\Im}_{R} \neq \mathfrak{\Im}$ then there is some nonzero pure state on $\mathfrak{\Im}$ by Proposition 1.4. Thus Theorem 2.3 shows that there is a pure state on $\mathfrak{U}$ which does not vanish on $\Im$. This contradicts Proposition 1.4 .

3. $U^{*}$-algebras. Although our primary interest is in Banach *-algebras it seems difficult to give the following proof in that setting without using the (more general) structure of $U^{*}$-algebras.

THEOREM 3.1. Let $\mathfrak{X}$ be a $U^{*}$-algebras. Let $\mathfrak{\Im}$ be $a{ }^{*}$-ideal of भ. Let $T$ be $a^{*}$-representation of $\mathfrak{\Im}$. Then there is a*-representation $\bar{T}$ of $\mathfrak{N}$ on $\left[\mathfrak{S}_{T}\right]$ which extends $T$. If $T$ is essential then $\bar{T}$ is unique, and the set of topologically cyclic vectors for T equals the set of topologically cyclic vectors for $\bar{T}$. Thus when $T$ is essential it is topologically cyclic or topologically irreducible iff $\bar{T}$ has the corresponding property.

Proof. If $T$ is not essential it is the direct sum of a zero sub*-representation $T^{0}$ on $\mathscr{S}_{0}$ and an essential sub-*-representation $T^{1}$ on $\mathfrak{S}_{1}$. We can extend $T^{0}$ as a zero ${ }^{*}$-representation $\bar{T}^{0}: \mathfrak{N} \rightarrow\left[\mathfrak{S}_{0}\right]$. Thus if we can extend $T^{1}$ to $\bar{T}^{1}: \mathfrak{U} \rightarrow\left[\mathscr{F}_{1}\right]$ then $T^{0} \oplus T^{1}$ extends $T$. Therefore we need only consider the case of essential *-representations.

Suppose $T$ is essential and let $\mathfrak{X}$ be the subset of $\mathfrak{F}_{2}, T_{\mathfrak{J}} \mathfrak{F}_{T}=$ $\left\{T_{b} x: b \in \mathfrak{S}, x \in \mathfrak{S}_{T}\right\}$. Then $\mathfrak{X}$ is dense in $\mathfrak{S}_{T}$. Let $\bar{T}: \mathfrak{A} \rightarrow\left[\mathfrak{S}_{T}\right]$ by any *-representation which extends $T$. Let $a \in \mathfrak{X}$ and $x \in \mathfrak{X}$. Then $x=$ $T_{b} y$ for $b \in \mathfrak{F}$ and $y \in \mathfrak{S}_{T}$. Thus

$$
\bar{T}_{a} x=\bar{T}_{a} T_{b} y=\bar{T}_{a} \bar{T}_{b} y=\bar{T}_{a b} y=T_{u b} y .
$$

Since $T$ is normed (Corollary 1.9) and $\mathfrak{X}$ is dense this shows that there is at most one extension $\bar{T}: \mathfrak{U} \rightarrow\left[\tilde{\mathcal{B}}_{T}\right]$ of $T$.

Suppose $z$ is a topologically cyclic vector for $T$. Let $\mathfrak{X}=T_{\Im} z$. Then $\mathfrak{X}$ is dense again. For $\alpha \in \mathfrak{X}$ and $x \in \mathfrak{X}$ define $\bar{T}_{a}^{1} x=T_{a b} z$ where $x=T_{b} z$ with $b \in \mathfrak{\Im}$. We must first show that this is well defined. Suppose $x=T_{d} z$ with $d \in \mathfrak{\Im}$ also. Let $a=\sum_{n=1}^{N} \lambda_{n} v_{n}$ where $\lambda_{n} \in C, v_{n} \in \mathfrak{A}_{q U}$, and $\sum_{n=1}^{N} \lambda_{n}=0$. Then $T_{a b} z-T_{a d} z=\sum_{n=1}^{N} \lambda_{m}\left(T_{v_{n} l-b} z-\right.$ $\left.T_{v_{n} d-d} z\right)$. However for each $n$

$$
\begin{aligned}
& \left\|T_{v_{n} b-b} z-T_{v_{n} d-d} z\right\|^{2}=\left\|T_{v_{n}(b-d)-(b-d)} z\right\|^{2} \\
& \quad=\left(\left(T_{(b-d) *\left(v_{n}^{*} v_{n}-v_{n}-v_{n}^{*}\right)(b-d)}+T_{(b-d) *(b-d)}\right) z, z\right) \\
& \quad=\left\|T_{b-d} z\right\|^{2}=0 .
\end{aligned}
$$

Thus $T_{a \zeta} z=T_{a \vdots} z$ and $\bar{T}_{a}^{1} x$ is well defined for each $x \in \mathfrak{X}$. For $a \in \mathfrak{\Im}$ 
and $x=T_{b} z \in \mathfrak{X}, \bar{T}_{a}^{1} x=T_{a b} z=T_{a} T_{b} z=T_{a} x$. It is easy to check that $\bar{T}^{1}$ is a ${ }^{*}$-representation of $\mathfrak{A}$ on the pre-Hilbert space $\mathfrak{X}$. Corollary 1.9 shows that $\bar{T}^{1}$ is normed and hence can be extended (in the sense of extensions of ${ }^{*}$-representations) to a unique *-representation $\bar{T}: \mathfrak{N} \rightarrow$ [ $\left.\mathfrak{S}_{T}\right]$ which extends $T: \mathfrak{\Im} \rightarrow\left[\mathfrak{S}_{T}\right]$. Clearly $z$ is a topologically cyclic vector for $\bar{T}$ since $\bar{T}_{\mathfrak{x}} z \supseteqq T_{3} z$. This concludes the proof of the theorem when $T$ is topologically cyclic.

Suppose $T$ is essential but not necessarily topologically cyclic. Then $T=\bigoplus_{\alpha \in A} T^{\alpha}$ is the internal direct sum of a family $\left\{T^{\alpha}: \alpha \in A\right\}$ of topologically cyclic sub-*-representations on $T$-invariant subspaces $\left\{\tilde{F}_{\alpha}: \alpha \in A\right\}$. For each $\alpha \in A$ we have shown how to construct a *representation $\bar{T}^{\alpha}: \mathfrak{A} \rightarrow\left[\mathfrak{S}_{\alpha}\right]$ which extends $T^{\alpha}: \mathfrak{\Im} \rightarrow\left[\mathscr{S}_{\alpha}\right]$. The direct sum $\bigoplus_{\alpha \in A} \bar{T}_{\alpha}: \mathfrak{A} \rightarrow\left[\mathfrak{S}_{T}\right]$ is defined since $\gamma_{\mathfrak{N}} \leqq \mathcal{V}_{\mathfrak{N}}$ by Corollary 1.11. It extends $T: \mathfrak{F} \rightarrow\left[\mathfrak{S}_{T}\right]$. We have already shown that only one such extension is possible. Thus any essential *-representation of $\mathfrak{\Im}$ has a unique extension to $\mathfrak{2}$.

Suppose $z$ is a topologically cyclic vector for $\bar{T}$ and $T$ is essential then $\bar{T}_{a} T_{b} z=T_{a b} z$ for all $a \in \mathfrak{Y}$ and $b \in \mathfrak{F}$ so that $T_{\Im} z^{-}$is a closed $\bar{T}$-invariant subspace of $\mathfrak{S}_{T}$ containing $z$ by [10, p. 206] or [9, 1.2.10]. The topological cyclicity of $z$ for $\bar{T}$ shows that $T_{\Im} z^{-}=\mathfrak{S}_{T}$ so that $z$ is a topologically cyclic vector for $T$.

When $T$ is essential we have shown that the set of topologically cyclic vectors for $\bar{T}$ equals the set of topologically cyclic vectors for $T$. Since a *-representation is topologically cyclic iff its set of topologically cyclic vectors is nonempty and is topologically irreducible iff every nonzero vector is topologically cyclic this establishes the last sentence of the theorem.

CoRollary 3.2. If $\mathfrak{X}$ is a $U^{*}$-algebra and $\mathfrak{\Im}$ is a ${ }^{*}$-ideal of $\mathfrak{A}$ included in $\mathfrak{U}_{R}$ then $\mathfrak{\Im}_{R}=\mathfrak{\Im}$. In particular $\left(\mathfrak{A}_{R}\right)_{R}=\mathfrak{U}_{R}$.

Proof. If $\mathfrak{\Im}_{R} \neq \mathfrak{\Im}$ there is a nonzero *-representation $T$ of $\mathfrak{\Im}$. Then Theorem 3.1 shows that there is a *-representation $\bar{T}$ of $\mathfrak{A}$ which does not vanish on $\mathfrak{\Im} \subseteq \mathfrak{U}_{R}$. This contradicts the definition of $\mathfrak{U}_{R}$.

Corollary 3.3. If $\mathfrak{2}$ is a $U^{*}$-algebra and $\mathfrak{\Im}$ is any *ideal of $\left(\mathfrak{U}_{R}\right)^{U}$ then $\mathfrak{\Im}_{R}=\mathfrak{\Im}$. In particular $\left(\left(\mathfrak{U}_{R}\right)^{U}\right)_{R}=\left(\left(\left(\mathfrak{U}_{R}\right)^{U}\right)_{R}\right)^{U}=\left(\mathfrak{U}_{R}\right)^{U}$.

Proof. The last sentence of Lemma 1.7 and Corollary 3.2 together show that $\left(\left(\mathfrak{U}_{R}\right)^{U}\right)_{R}=\left(\mathfrak{U}_{R}\right)^{U}$. Thus these sets clearly equal $\left(\left(\left(\mathfrak{U}_{R}\right)^{U}\right)_{R}\right)^{U}$. Thus this corollary follows from Corollary 3.2 applied to $\left(\mathfrak{U}_{R}\right)^{U}$ in place of $\mathfrak{A}$.

Corollary 3.4. If $\mathfrak{A}$ is a Banach *-algebra and $\mathfrak{I}$ is $a{ }^{*}$-ideal 
of $\mathfrak{U}_{R}$ then $\mathfrak{\Im}_{R}=\mathfrak{\Im}$. In particular $\left(\mathfrak{A}_{R}\right)_{R}=\mathfrak{A}_{R}$.

Proof. Theorem 1.12 and Proposition 1.4 together show that $\mathfrak{A}$ and $\mathfrak{A}_{R}$ are $U^{*}$-algebras so that $\mathfrak{A}_{R}=\left(\mathfrak{A}_{R}\right)^{U}$. Thus this corollary follows from Corollary 3.3.

4. Remarks on categorical consequences. In this section we wish to indicate the consequences of our results in the language of categories. In reference [3] we find a strong notion of radical subcategory which we will use. In fact what is called a radical in [3] is sometimes called a hereditary radical (cf. p. 125 of N. J. Divinsky, Rings and Radicals, University of Toronto Press, 1965). From one viewpoint our results may be considered as a quite different example of this theory.

We will show first that each of the three categories listed in the introduction is both semi-abelian and co-semi-abelian. The trivial *-algebra $\{0\}$ is a zero-object in each of these categories and also in each of the other categories which we will consider. We examine the categorically defined kernels, cokernels, images, and co-images in these categories.

In all three of the categories listed in the introduction the kernel of $f \in \operatorname{Hom}(\mathfrak{A}, \mathfrak{B}$ ) is simply (the subobject represented by the injection into $\mathfrak{A}$ of) the set theoretic kernel $\operatorname{Ker}(f)$ of $f$.

Consider the following categories.

(4) $U^{*}$-algebras and *-homomorphisms.

(5) Banach *-algebras and *-homomorphisms.

Since the image of any $U^{*}$-algebra is a $U^{*}$-algebra it is easy to see that the kernel of $f \in$ Hom (IV, $\mathfrak{B}$ ) in category (4) is (the subobject represented by the injection into $\mathfrak{A}$ of) $(\operatorname{ker}(f))^{U}$ where again $\operatorname{Ker}(f)$ is the set theoretic kernel of $f$. In category (5) morphisms do not always have kernels, since there is not in general any maximal subobject of $\operatorname{Ker}(f)$ on which a Banach *-algebra norm can be defined. Notice that when such a maximal subobject does exist it must be included in $(\operatorname{Ker}(f))^{U}$.

In the category

(6) *-algebras and *-homomorphisms

the set theoretic kernel "is" the categorical kernel.

In categories (1), (4) and (6) the cokernel of $f \in \operatorname{Hom}(\mathfrak{A}, \mathfrak{B})$ is represented by

$$
\left.\mathfrak{B} \longrightarrow \mathfrak{B} / \text { * }^{*} \text {-ideal generated by } f(\mathfrak{C})\right) \text {. }
$$

In categories (2) and (3) the cokernel of $f \in \mathrm{Hom}(\mathfrak{A}, \mathfrak{B})$ is represented by

$$
\mathfrak{B} \longrightarrow(\mathfrak{B} /(\text { closed *-ideal generated by } f(\mathfrak{U}))) \text {. }
$$


Morphisms in category (5) do not always have cokernels, since there is not always a smallest *-ideal containing $f(\mathfrak{U})$ such that the quotient may be embedded in a Banach *-algebra.

In categories (1), (2), (3), (4), (6) the image of $f \in \mathrm{Hom}(\mathfrak{U}, \mathfrak{B})$ is represented by the map

$$
\mathfrak{X} / \operatorname{Ker}(f) \longrightarrow \mathfrak{B}
$$

induced by $f$. Morphisms in category (5) do not always have images. The co-image of $f \in \operatorname{Hom}(\mathfrak{A}, \mathfrak{B})$ in categories (1), (2), (3), (4), and (6) is represented by the natural morphism

$$
\mathfrak{A} \longrightarrow \mathfrak{A} / \operatorname{Ker}(f) \text {. }
$$

Morphisms in category (5) do not always have co-images.

DEFINITION 4.1. A category with a zero object is called semiabelian if:

(a) Every morphism may be factored into a representative of its co-image followed by a representative of its image, and

(b) Every morphism has a cokernel.

A category with a zero object is called co-semi-abelian iff it satisfies (a) and

(c) Every morphism has a kernel.

Proposition 4.2. Categories (1), (2), (3), (4), and (6) are each both semi-abelian and co-semi-abelian.

Proof. This follows from the remarks above.

Definition 4.3. Let $\mathscr{C}$ be a semi-abelian category. A radical subcategory of $\mathscr{C}$ is a full subcategory $\mathscr{R}$ such that

(a) If $\mathfrak{A} \in \mathscr{R}, f \in \operatorname{Hom}(\mathfrak{A}, \mathfrak{B})$ and $i \in \operatorname{Hom}(\mathfrak{\Im}, \mathfrak{B})$ represents the image of $f$ then $\Im \in \mathscr{R}$.

(b) If $\mathfrak{X} \in \mathscr{R}, f \in \operatorname{Hom}(\mathfrak{R}, \mathfrak{B})$ and $k \in \operatorname{Hom}(\mathfrak{R}, \mathfrak{Q})$ represents the kernel of $f$ then $\Re \in \mathscr{R}$.

(c) For each $\mathfrak{A} \in \mathscr{C}$ there is a unique subobject $\mathfrak{A}_{\mathscr{R}}$ or $\mathfrak{A}$ which satisfies

$\left(c_{1}\right) \quad \mathfrak{U}_{\mathscr{B}}$ is a kernel.

$\left(c_{2}\right) \mathfrak{U}_{\mathscr{A}}$ is represented by a monomorphism with an object of $\mathscr{R}$ as domain.

$\left(c_{3}\right) \mathfrak{U}_{\mathscr{A}}$ includes any subobject of $\mathfrak{U}$ which is a kernel and is also represented by a monomorphism with an object of $\mathscr{R}$ as domain.

(d) If $u \in \mathrm{Hom}(\mathfrak{A}, \mathfrak{B})$ is a representative of the cokernel of a representative $v \in \operatorname{Hom}(\mathfrak{F}, \mathfrak{U})$ of $\mathfrak{U}_{\mathscr{A}}$ then the subobject $\mathfrak{B}_{\mathscr{A}}$ is the zero-subobject of $\mathfrak{B}$. 
THEOREM 4.4. In each of the categories (1), (2), and (3) the full subcategory defined by the class of objects $\mathfrak{X}$ such that $\mathfrak{A}=\mathfrak{U}_{R}$ is a radical subcategory.

Proof. Proposition 1.4 and Corollary 2.4 and 3.4, together with the identification of the kernels, cokernels, images and co-images in these categories, establish this result.

This theorem justifies the term *-radical as a name for the reducing ideal in these three categories.

In the semi-abelian category (4) of $U^{*}$-algebras we do not know whether the reducing ideal is always a $U^{*}$-algebra, i.e.

$$
\mathfrak{A}_{R}=\left(\mathfrak{U}_{R}\right)^{U} \text {. }
$$

In fact we do not know whether every closed *-ideal is always a $U^{*}$-algebra. If $\mathfrak{X}_{R}$ is always a $U^{*}$-algebra then Theorem 4.4 is true for category (4) also. Otherwise one might consider the full subcategory $\mathscr{R}$ defined by the class of objects $\mathfrak{U}$ such that $\mathfrak{X}=\left(\mathfrak{U}_{R}\right)^{U}$. This subcategory satisfies (a), (b), and (c) of Definition 4.3 with $\mathfrak{U}_{\mathscr{R}}=\left(\mathfrak{\Re}_{R}\right)^{U}$. However it will not satisfy (d) unless

$$
\left(\left(\mathfrak{U} /\left(\mathfrak{P}_{R}\right)^{U}\right)_{R}\right)^{U}=\{0\} \text {. }
$$

It is possible that condition (4.2) is true for all $U^{*}$-algebras. If it is not true for all $U^{*}$-algebras perhaps there is a full subcategory of category (4) in which either condition (4.1) or (4.2) holds. This subcategory might have a radical subcategory associated with the reducing ideal. Notice that categories (2) and (3) are nonfull subcategories of category (4) in which (4.1) holds.

It seems unlikely that the semi-abelian category (6) has a radical subcategory defined by the reducing ideal. However a counterexample is probably quite weird. (Note added in proof: I have found a counterexample which is not particularly weird.)

\section{REFERENCES}

1. J. Dixmier, Les $C^{*}$-algebras et leurs Representations, Gauthier-Villars, Paris (1964).

2. I. Gelfand and M. Naimark, Normed rings with involution and their representations, Izvestiya Akademii Nauk S.S.S.R. Ser. Mat., 12 (1948), 445-480.

3. M. Gray, A Radical Approach to Algebra, Addison Wesley, Reading, Mass., (1970).

4. B. E. Johnson, An introduction to the theory of centralizers, Proc. London Math. Soc., (3) 14 (1964), 299-320.

5. J. L. Kelley and R. L. Vaught, The positive cone in Banach algebras, Trans. Amer. Math. Soc., 74 (1953), 44-55.

6. M. A. Naimark, Normed Rings, Noordhoff, Groningen, 1964. 
7. T. W. Palmer, The Gelfand-Naimark pseudo-norm on Banach*-algebras, J. London Math. Soc., (2) 2 (1970), 89-96.

8. - *-Representations of $U^{*}$-algebras, Indiana University Math. J., 20 (1971), 929-933.

9. T. W. Palmer, *-Algebras, in preparation.

10. C. E. Rickart, General Theory of Banach Algebras, Van Nostrand, Princeton, 1960. 11. B. Yood, Faithful *-representations of normed algebras II, Pacific J. Math. 14 (1964), 1475-1487.

Received March 30, 1971.

UNIVERSITY OF OREGON 



\section{PACIFIC JOURNAL OF MATHEMATICS}

\section{EDITORS}

\section{H. SAMElson}

Stanford University

Stanford, California 94305

C. R. Новву

University of Washington

Seattle, Washington 98105
J. DugunduI

Department of Mathematics University of Southern California

Los Angeles, California 90007

RICHARD ARENS

University of California

Los Angeles, California 90024

\section{ASSOCIATE EDITORS}
E. F. BECKENBACH
B. H. NeUmanN
F. WOLF
K. YosHIDA

\section{SUPPORTING INSTITUTIONS}

UNIVERSITY OF BRITISH COLUMBIA

UNIVERSITY OF SOUTHERN CALIFORNIA

CALIFORNIA INSTITUTE OF TECHNOLOGY

STANFORD UNIVERSITY

UNIVERSITY OF CALIFORNIA

UNIVERSITY OF TOKYO

MONTANA STATE UNIVERSITY

UNIVERSITY OF UTAH

UNIVERSITY OF NEVADA

WASHINGTON STATE UNIVERSITY

NEW MEXICO STATE UNIVERSITY

OREGON STATE UNIVERSITY

UNIVERSITY OF OREGON

OSAKA UNIVERSITY

UNIVERSITY OF WASHINGTON

* *

AMERICAN MATHEMATICAL SOCIETY

NAVAL WEAPONS CENTER 


\section{Pacific Journal of Mathematics}

\section{Vol. 43, No. $1 \quad$ March, 1972}

Alexander (Smbat) Abian, The use of mitotic ordinals in cardinal

arithmetic ....................................... 1

Helen Elizabeth. Adams, Filtrations and valuations on rings ......... 7

Benno Artmann, Geometric aspects of primary lattices .............. 15

Marilyn Breen, Determining a polytope by Radon partitions ........... 27

David S. Browder, Derived algebras in $L_{1}$ of a compact group .......... 39

Aiden A. Bruen, Unimbeddable nets of small deficiency .............. 51

Michael Howard Clapp and Raymond Frank Dickman, Unicoherent

compactifications ............................... 55

Heron S. Collins and Robert A. Fontenot, Approximate identities and the strict topology ................................... 63

R. J. Gazik, Convergence in spaces of subsets................. 81

Joan Geramita, Automorphisms on cylindrical semigroups ........... 93

Kenneth R. Goodearl, Distributing tensor product over direct product ..... 107

Julien O. Hennefeld, The non-conjugacy of certain algebras of

operators ................................... 111

C. Ward Henson, The nonstandard hulls of a uniform space ........... 115

M. Jeanette Huebener, Complementation in the lattice of regular

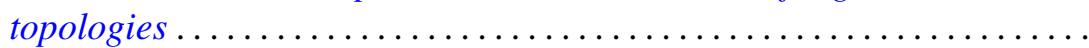

Dennis Lee Johnson, The diophantine problem $Y^{2}-X^{3}=A$ in a

polynomial ring .................................... 151

Albert Joseph Karam, Strong Lie ideals . . . . . . . . . . . . . . . . . . . . 157

Soon-Kyu Kim, On low dimensional minimal sets ............... 171

Thomas Latimer Kriete, III and Marvin Rosenblum, A Phragmén-Lindelöf

theorem with applications to $M(u, v)$ functions ..... . .

William A. Lampe, Notes on related structures of a universal algebra . . . . 189

Theodore Windle Palmer, The reducing ideal is a radical .

207

Kulumani M. Rangaswamy and N. Vanaja, Quasi projectives in abelian and module categories ................................ 221

Ghulam M. Shah, On the univalence of some analytic functions ......... 239

Joseph Earl Valentine and Stanley G. Wayment, Criteria for Banach

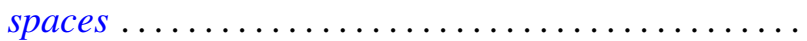

Jerry Eugene Vaughan, Linearly stratifiable spaces ............... 253

Zbigniew Zielezny, On spaces of distributions strongly regular with respect to partial differential operators ..................... 